\title{
Capacity and Outage Probability Analysis of Network Coding based User Cooperation Transmission
}

\author{
Yinglei Teng Mei Song Yong Zhang Yuanyuan Liu Junde Song \\ Beijing University of Posts and Telecommunications \\ Beijing, China \\ lilytengtt@gmail.com songm@bupt.edu.cn yongzhang@bupt.edu.cn lyy886200@bupt.cn jdsong@bupt.edu.cn
}

\begin{abstract}
The two user cooperative transmission context coincides with symmetric feature of network coding (NC), which makes it possible to perform distributed random coding, thus exploring both the cooperative diversity and the network coding diversity. In this paper, we provide performance analysis of network coding based two-user cooperation under an OFDMA based orthogonal physical layer channel, in terms of the channel capacity and outage probability. Expressions for exact capacity are devised and closed form of outage probability with lower bound is presented. Compared with the conventional user cooperation transmission, the $\mathrm{NC}$ based two-user cooperation brings $11.8 \%$ higher capacity and $50 \%$ lower outage probability.
\end{abstract}

Keywords-User cooperation; network coding; OFDMA; link capacity; outage probability;

\section{INTRODUCTION}

The concept of cooperative transmission has been well recognized as an effective countermeasure against multi-path fading by exploring the cooperative diversity instead of the traditional multi-antenna diversity. Actually, both the relay infrastructures and peer users in the network can act as the cooperative partners.

Placing a relay station in the network allows a BS to cover the shadowed areas and to extend the limited coverage range [1]. However, it increases the deployment costs, introduces a high management complexity. Meanwhile, user cooperation is more flexible. It brings a wide robustness and makes any opportunity of relay radios or diversity gains by occasional cooperation transmission. Such user cooperative scenario has been found in LMDS/MMDS mesh networks [2], IEEE802.16j [3], and so on.

In order to improve the cooperative gains, [4] proposed an algebraic superposition of channel codes. Using the concept of network coding, two source nodes paired as partners to encode together and deliver the composite packets to a common destination. The combination of network coding with user cooperative transmission stands out for mainly two reasons: first, the broadcast nature of the wireless channel allows for the exploitation of some of the particular features of network coding [5]; second, the user cooperative transmission context coincides with symmetric feature of $\mathrm{NC}$, which makes it possible to perform distributed random coding.

There have been investigations of performance analysis of the network coding approaches to the cooperative diversity; however, most of the work gives only integral-form outage probability or ergodic capacity [6-9], while no closed formulation was devised. Besides, few works were done involved with the OFDMA based physical layer channel. Therefore, focusing on the transmission performance, we analyze the user cooperative transmission with and without NC elaborately in this paper. There are some outstanding contributions: first, the application of OFDMA technology provides the orthogonal channels for the two cooperative users, which makes the duplex transmission feasible. Second, the closed formulations of link capacity with network coding are pioneered. Third, the outage probability for such cooperation scenario with $\mathrm{NC}$ is particularly deduced basing on the OFDMA channels.

The remaining sections of the paper are organized as follows. Section II presents the channel assumption and transmission model of the two user cooperation scenario with and without NC. Section III studies two information theoretic metrics to evaluate the performance of the NC_based user cooperative transmission. Section IV is devoted to present the simulation result and performance evaluation. Finally, section $\mathrm{V}$ concludes the paper.

\section{TRANSMISSION MODEL AND MATHEMATICAL FORMULATION}

\section{A. OFDMA Channel Model}

We adopt OFDMA as an underlying physical layer technique, where users are assigned with subcarriers either by maximizing channel gain or other fairness considered approaches; hence, the two cooperating nodes communicate independent information over orthogonal channels.

Consider a simple two user scenario to share $N$ subcarriers. Denote $\mathcal{N}$ the system subcarrier set and $|\mathcal{N}|=N$. Assume that channel gain from transmitter $i(i \in\{A, B\})$ to receiver $j$ $(j \in\{B, A\})$ on subcarrier $n$ is $h_{(i, j), n}$, which is identically distributed (i.i.d) zero-mean complex variables of symmetric Gaussian distribution with variance $\sigma_{h}^{2}$ on all links, writes as $C N \in\left(0, \sigma_{h}^{2}\right)$. Meanwhile, the additive white Gaussian noise $n_{i, n}$ with the distribution of $C N \in\left(0, \sigma_{n}^{2}\right)$. Let $D_{(i, j), n}$ Corresponds to the frequency-domain channel fading coefficient between the transmitter $i$ and receiver $j$ on 
subcarrier $n$. Since $D_{(i, j), n}$ is independent for every user, $D_{(i, j), n} \quad$ satisfies distribution $\quad C N \in\left(0, \sigma_{d}^{2}\right) \quad$ where $\sigma_{d}^{2}=N \sigma_{h}^{2}[12]$.

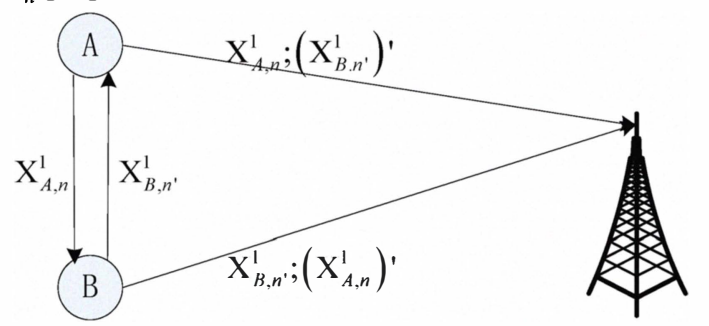

(a)

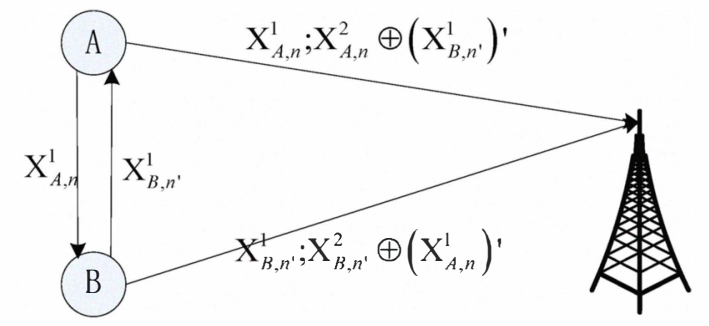

(b)

Figure 1. Two transmission models of user cooperation

\section{B. Cooperative Transmission Protocal and Formulation}

In the user cooperation transmission, each user can work as the source as well as a potential relay. Such symmetric system model provides opportunity for users to obtain cooperation diversity from others. A symmetric cooperative communication system model consists of two user nodes, i.e, A, B, and a BS, indexed as $s$ in the formulation, is pictured in Fig.1, where (a) shows the conventional cooperation model while (b) gives a $\mathrm{NC}$ _based cooperation model.

In the first time slot, users A and B broadcast separate source data $x_{A, n}^{1}, x_{B, n^{\prime}}^{1}$ on their own subcarriers $n$ and $n^{\prime}$. Denote $\mathcal{N}_{a}, \mathcal{N}_{b}$ as corresponding subcarrier sets for them. In the second time slot, users working as pure forwarding relays, transmit $\left(x_{B, n}^{1}\right)^{\prime},\left(x_{A, n^{\prime}}^{1}\right)^{\prime}$ on the same channels, as depicted in Fig.1-(a); while in Fig.1-(b), both users transmit a combined coding data of $x_{A, n}^{2} \oplus\left(x_{B, n}^{1}\right)^{\prime}$ and $x_{B, n^{\prime}}^{2} \oplus\left(x_{A, n}^{1}\right)^{\prime}$. Herein, $\left(x_{A, n}^{1}\right)^{\prime}$, $\left(x_{B, n^{\prime}}^{1}\right)^{\prime}$ are forwarding data, which is identical or amplified depending on different forwarding schemes, i.e., DF and AF [10]. Note that the channel allocation for each user is assumed to be constant over the two time slots, denoted as $h_{(A, s), n}$, $h_{(B, s), n}$ and $h_{(i, j), n}$, and for simplification, $h_{(A, s), n}, h_{(B, s), n}$ are written as $h_{A, n}, h_{B, n}$. Note also that we take the superscript as the time index, and subscript as the user and subcarrier index throughout this paper. Besides, we assume the perfect channel state information (CSI) in the following analysis.

\section{INFORMATION THEORETIC ANALYSIS: CAPACITY AND OUTAGE PROBABILITY}

In this section, we aim to study two different information theoretic metrics to evaluate the performance of the NC_based user cooperative transmission. In what follows, the expression of the two kinds of system metrics is developed for the NC_based cooperation transmission (CT_NC) compared with the conventional cooperation transmission (CT) and direct transmission (DT).

\section{A. Capacity Derivation}

For the sake of transmission formulation, let $y_{A, n}^{1}, y_{B, n^{\prime}}^{1}$ be the received vector of symbols by user $A$ and $B$ in the first time slot, while $y_{A, n}^{2}, y_{B, n^{\prime}}^{2}$ is the received symbol in the second time slot. Three different transmissions within two time slot can be formulated as follows:

In the DT case, messages are transmitted directly from user to destination without help from any external source. Thereby, the channel capacity for one user $R_{i}^{D T}$ in the two time slots is defined

$r_{i, n}^{D T}=2 \cdot \log _{2}\left(1+\frac{\left|h_{i, n}\right|^{2} p_{i, n}}{\Gamma \sigma^{2}}\right)$

$R_{i}^{D T}=\sum_{n=1}^{N} \rho_{i, n} r_{i, n}^{D T}$

where $r_{i, n}^{D T}$ is the rate on subcarrier $n, \rho_{i, n}$ is the subcarrier allocation indicator. It equals to 1 when the subcarrier is assigned to the $i^{t h}$ user and 0 otherwise, and they are constant within one scheduling time. $\Gamma=\frac{-1.5}{\ln \left(5 B_{k}^{\min }\right)}$ is the SNR gap related to a minimal targeted bit-error-ratio $B_{k}^{\min }$.

In the CT case as described in the Fig.1-(a), taking the DF as the forwarding strategies, the reception signal can be formulated as:

Slot 1st:

$\left(\begin{array}{l}y_{A, n}^{1} \\ y_{B, n^{\prime}}^{1}\end{array}\right)=\left(\begin{array}{cc}h_{A, n} \sqrt{p_{A, n}} & 0 \\ 0 & h_{B, n^{\prime}} \sqrt{p_{B, n^{\prime}}}\end{array}\right)\left(\begin{array}{l}x_{A, n}^{1} \\ x_{B, n^{\prime}}^{1}\end{array}\right)+\left(\begin{array}{c}n_{A, n}^{1} \\ n_{B, n^{\prime}}^{1}\end{array}\right)$

Slot 2nd:

$\left(\begin{array}{c}y_{A, n}^{2} \\ y_{B, n^{\prime}}^{2}\end{array}\right)=\left(\begin{array}{cc}h_{B, n^{\prime}} \sqrt{p_{B, n^{\prime}}} & 0 \\ 0 & h_{A, n} \sqrt{p_{A, n}}\end{array}\right)\left(\begin{array}{c}x_{A, n}^{1} \\ x_{B, n^{\prime}}^{1}\end{array}\right)+\left(\begin{array}{c}n_{A, n}^{1} \\ n_{B, n^{\prime}}^{1}\end{array}\right)$

where $p_{A, n}, p_{B, n^{\prime}}$ are the transmit power from user $\mathrm{A}$ and $\mathrm{B}$ respectively on the subcarrier $n$ and $n^{\prime}$. Note that we assume that the transmit power holds still over the two time slots. Let $H_{i, n n^{\prime}}$ means the cooperative equivalent channel of use $i$ on subcarrier $n$ and $n$ '. The link capacity of user $i$ on the $n$ subcarrier and $n$ ' relaying subcarrier $r_{i, n n^{\prime}}^{C T}$ is combined as [11]: 


$$
\begin{aligned}
& r_{i, n n^{\prime}}^{C T}=\log _{2}\left(\operatorname{det}\left(I+H_{i, n n^{\prime}} \cdot H_{i, n n^{\prime}}^{H} / \Gamma \sigma^{2}\right)\right) \\
& =\log _{2}\left(1+\rho_{i, n} \frac{\left|h_{i, n}\right|^{2} p_{i, n}}{\Gamma \sigma^{2}}+\sum_{j \neq i} \rho_{j, n^{\prime}} \frac{\left|h_{j, n^{\prime}}\right|^{2} p_{j, n^{\prime}}}{\Gamma \sigma^{2}}\right) \\
& R_{i}^{C T}=\sum_{n=1}^{N} r_{i, n n^{\prime}}^{C T}
\end{aligned}
$$

In the CT_NC case as described in the Fig.1-(b), the reception signal in the first time slot is the same with that in $\mathrm{CT}$, but in the second time slot, the reception is expressed as:

$$
\begin{aligned}
& y_{A, n}^{2}=h_{A, n} \sqrt{p_{A, n}}\left(x_{A, n}^{2} \oplus x_{B, n^{\prime}}^{1}\right)+n_{A, n}^{2} \\
& y_{B, n^{\prime}}^{2}=h_{B, n^{\prime}} \sqrt{p_{B, n^{\prime}}}\left(x_{B, n^{\prime}}^{2} \oplus x_{A, n}^{1}\right)+n_{B, n^{\prime}}^{2}
\end{aligned}
$$

Herein, $\oplus$ is the bit-wise XOR operator. Assuming that at the destination, cyclic redundancy check (CRC) is supposed to detect decoding error; and by individual decoding, each source's information bits are recovered by decoding a packet which is formed by code combining of this source's packet received in the first phase and the network-coded packet [9]. In fact, if $x_{A, n}^{1}$ and $x_{B, n}^{1}$ is received and decoded correctly at $\mathrm{T} 1$ slot, then in the next slot, the receiver can recover each source's bits $x_{A, n}^{2}$ and $x_{B, n^{\prime}}^{2}$ by individual or joint decoding from the network-coded packets $\left(x_{A, n}^{2} \oplus x_{B, n^{\prime}}^{1}\right)$ and $\left(x_{B, n^{\prime}}^{2} \oplus x_{A, n}^{1}\right)$. Meanwhile, the network-coded packets with $x_{B, n^{\prime}}^{1}$ and $x_{A, n}^{1}$ component are used as incrementally redundant information as these packets are received over two paths equivalently.

In order to deduct capacity, we introduce a coding vector $\mathbf{C}$, where $\left|c_{A, n}\right|^{2}=\left|c_{B, n}\right|^{2}=1$, then (5) can be rewritten as:

$$
\begin{aligned}
y_{A, n}^{2} & =h_{A, n} \sqrt{p_{A, n}} x_{A, n}^{2} \oplus h_{A, n} \sqrt{p_{A, n}} x_{B, n^{\prime}}^{1}+n_{A, n}^{2} \\
& =h_{A, n} \sqrt{p_{A, n}} x_{A, n}^{2}+c_{A, n} h_{A, n} \sqrt{p_{A, n}} x_{B, n^{\prime}}^{1}+n_{A, n}^{2} \\
y_{j, n^{\prime}}^{2} & =h_{B, n^{\prime}} \sqrt{p_{B, n^{\prime}}} x_{B, n^{\prime}}^{2} \oplus h_{B, n^{\prime}} \sqrt{p_{B, n^{\prime}}} x_{A, n}^{1}+n_{B, n^{\prime}}^{2} \\
& =h_{B, n^{\prime}} \sqrt{p_{B, n^{\prime}}} x_{B, n^{\prime}}^{2}+c_{B, n^{\prime}} \cdot h_{B, n^{\prime}} \sqrt{p_{B, n^{\prime}}} x_{A, n}^{1}+n_{B, n^{\prime}}^{2}
\end{aligned}
$$

Accordingly, the received signal for two users over the whole cooperative period can be expressed as

$$
\left(\begin{array}{l}
y_{A, n}^{1} \\
y_{B, n^{\prime}}^{1} \\
y_{A, n}^{2} \\
y_{B, n^{\prime}}^{2}
\end{array}\right)=\mathbf{H}\left(\begin{array}{c}
x_{A, n}^{1} \\
x_{B, n^{\prime}}^{1} \\
x_{A, n}^{2} \\
x_{B, n^{\prime}}^{2}
\end{array}\right)+\left(\begin{array}{c}
n_{A, n}^{1} \\
n_{B, n^{\prime}}^{1} \\
n_{A, n}^{2} \\
n_{B, n^{\prime}}^{2}
\end{array}\right)
$$

where

$$
\mathbf{H}=\left(\begin{array}{cccc}
h_{A, n} \sqrt{p_{A, n}} & 0 & 0 & 0 \\
0 & h_{B, n^{\prime}} \sqrt{p_{B, n^{\prime}}} & 0 & 0 \\
0 & c_{A, n} h_{A, n} \sqrt{p_{A, n}} & h_{A, n} \sqrt{p_{A, n}} & 0 \\
c_{B, n} h_{B, n^{\prime}} \sqrt{p_{B, n^{\prime}}} & 0 & 0 & h_{B, n^{\prime}} \sqrt{p_{B, n^{\prime}}}
\end{array}\right)
$$

Therefore, the link capacity of user $i$ on the $n$ subcarrier and $n^{\prime}$ relaying subcarrier $r_{i, n n^{\top}}^{C T C}$ in this mode is developed as: $r_{i, n n^{\prime}}^{C T}{ }^{N C}=\log _{2}\left(\operatorname{det}\left(I+H_{i, n n^{n}} \cdot H_{i, n n^{\prime}}^{H}, / \Gamma \sigma^{2}\right)\right)$

$=\log _{2}\left(\begin{array}{l}\left(1+2\left|A_{i, n}\right|^{2}+\sum_{j \neq i}\left|B_{j, n}\right|^{2}+\sum_{j \neq i}\left|A_{i, n}\right|^{2}\left|B_{j, n}\right|^{2}\right) \\ \cdot\left(1+\left|A_{i, n}\right|^{2}+\sum_{j \neq i} 2\left|B_{j, n}\right|^{2}+\sum_{j \neq i}\left|A_{i, n}\right|^{2}\left|B_{j, n}\right|^{2}\right)\end{array}\right)$

$R_{i}^{C T_{-} N C}=\sum_{n=1}^{N} r_{i, n n^{-N}}^{C T}$

where

$$
\begin{aligned}
& \left|A_{i, n}\right|^{2}=\rho_{i, n}\left|h_{i, n}\right|^{2} p_{i, n} / \Gamma \sigma^{2} \\
& \left|B_{j, n^{\prime}}\right|^{2}=\rho_{j, n^{\prime}}\left|h_{j, n^{\prime}}\right|^{2} p_{j, n^{\prime}} / \Gamma \sigma^{2}
\end{aligned}
$$

\section{B. Outage Probability Analysis}

Outage probability is termed to show robustness of a communication system and measures the data rate that can be supported with a certain error probability.

To derive the overall outage probability conditioned on the three cases: DT, CT, and CT_NC, we need to first give the outage probability of each OFDMA based link. Referring to [12], we define the subcarrier outage probability as:

$$
p_{s}=\operatorname{Pr}\left\{\log _{2}\left(1+\frac{\left|[D]_{D_{(i, j, n)}}\right|^{2} S N R}{\Gamma}\right)<R_{n}\right\}
$$

Herein, $R_{n}$ is the target transition rate on subcarrier $n$. Due to the variant fading and adaptive coding modulation (ACM) on each subcarrier of OFDMA system, $R_{n}$ may differ on different subcarriers. Define the upper bound when BPSK modulation is adapted, we have

$$
p_{s}=1-\exp \left(-\frac{2^{R_{n}}-1}{\sigma_{d}^{2}} \frac{1}{S N R}\right)
$$

When $p_{s}=S N R \rightarrow \infty$, we have

$$
p_{s} \approx \frac{2^{R_{n}}-1}{\sigma_{d}^{2}} \frac{1}{S N R}
$$

Assuming that the subcarrier allocation guarantees the outage probability is identical for every user, ${ }^{1}$ we define $N \geq M \geq 1$, and the user outage probability satisfies [13]:

$$
p_{u}=p_{s}^{N} f\left(p_{s}\right)
$$

where $f\left(p_{s}\right)$ is given by $f\left(p_{s}\right)=a_{0}+a_{1} p_{s}+\cdots+a_{(M-1) N} p_{s}^{(M-1) N}$, $a_{0} \neq 0$.

\footnotetext{
${ }^{1}$ The random bipartite graph model can be used to formulate the subcarrier allocation problem in OFDMA systems [12].
} 
Thereby, in the two user cooperative cases,

$$
P_{u}=p_{s}^{N}\left(a_{0}+a_{1} p_{s}+\cdots+a_{N} p_{s}^{N}\right)=a_{0} p_{s}^{N}+a_{1} p_{s}^{N+1}+\mathrm{O}\left(P_{s}^{N+1}\right)
$$

Meanwhile, according to approximation results for both high SNR and low SNR regime in [13], we obtain a complete picture of the outage performance for the OFDMA systems, when $N>2$, i.e., $a_{0}=1, a_{1}=0$. Hence, we get

$$
P_{u}=p_{s}^{N}
$$

Now we are ready to rigorously formulate our problem. We define the overall outage probability of various strategies discussed previously as either of the source date fail to be received successfully. That is to say the overall outage $p_{\text {out }}$ occurs when either of the two users fails to send out the source data successfully, namely,

$$
p_{\text {out }}=p_{A}+p_{B}-p_{A B}
$$

where $p_{A}$ and $p_{B}$ is the outage probability for user $\mathrm{A}$ and $\mathrm{B}$ on all links, $p_{A B}$ is the combined outage probability when $\mathrm{A}$ and $\mathrm{B}$ both fail.

Let's define the probability of DT, CT and CT NC cases as $p_{\text {out } 1}, p_{\text {out } 2}$ and $p_{\text {out } 2}$ respectively. Define the outage probability of the direct link from user A and $B$ to the destination in the Slot 1 as $p_{\text {out } a}, p_{\text {out } b}$, the outage probability of cooperative link in the Slot 2 as $p_{\text {out }} a^{\prime}, p_{\text {out }} b^{\prime}$. Assume that the subcarrier allocation guarantees the outage probability is identical for every user; meanwhile, assuming an upper bound of outage probability when BPSK modulation is adapted on each subcarrier, we have $p_{\text {out } a}=p_{\text {out }} b$ $=p_{\text {out } a^{\prime}}=p_{\text {out } b_{-} b^{\prime}}=p=p_{u}$. Accordingly, the system outage probability of three different transmissions cases within two time slot can be formulated as follows:

In the DT case, the overall outage of two users occurs when either of them fails to send out the source data successfully. That is,

$$
p_{A}=p_{\text {out_a }} ; \quad p_{B}=p_{\text {out }_{-} b} ; \text { and } p_{A B}=p_{A} p_{B},
$$

then

$$
p_{\text {out } 1}=p_{\text {out_ } a}+p_{\text {out } b}-p_{\text {out_a }} p_{\text {out } b}=2 p-p^{2}=2 p_{s}^{N}-p_{s}^{2 N}
$$

In the CT case, the outage event of one user occurs when both the data links, i.e., the direct link or the cooperative link, fails to send out the source data successfully. That is,

$$
p_{A}=p_{\text {out_a }} p_{\text {out_ } b^{\prime}} ; \quad p_{B}=p_{\text {out_a }} p_{\text {out_b }} ; \text { and } p_{A B}=p_{A} p_{B},
$$

then

$$
\begin{aligned}
& p_{\text {out } 2}=p_{\text {out } \_} p_{\text {out } b^{\prime}}+p_{\text {out } a_{-}} p_{\text {out_b } b}-p_{\text {out }_{-} a} p_{\text {out } b} \cdot p_{\text {out } a_{-}} p_{\text {out } b_{-} b^{\prime}} \\
& =2 p^{2}-p^{4}=2 p_{s}^{2 N}-p_{s}^{4 N}
\end{aligned}
$$

In the CT NC case, the outage event of one user occurs when both the direct links fail or only one direct success but both the cooperative links fail to send out the XOR operated packet data. That is,

$$
\begin{aligned}
& p_{A}=p_{\text {out } a} p_{\text {out } b}+p_{\text {out }_{-} a}\left(1-p_{\text {out } b}\right) p_{\text {out_a }} p_{\text {out } b_{-}} ;
\end{aligned}
$$

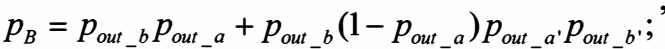

Meanwhile, the event of both source data failing to reach the destination happens when outage occurs on both the direct links, namely,

$$
p_{A B}=p_{\text {out_a }} p_{\text {out } b} b \text {, }
$$

then

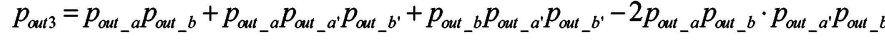

$$
\begin{aligned}
& =p^{2}+2 p^{3}-2 p^{4}=p_{s}^{2 N}+2 p_{s}^{3 N}-2 p_{s}^{4 N}
\end{aligned}
$$

\section{SimUlation RESUlTS}

In this section, we will provide detailed simulation results to compare the performance of user cooperative transmission with or without network coding. Note that the basic observing time window is 2 time slots for both metrics. The total bandwidth is $1 \mathrm{M}$ divided by $\mathrm{N}(\mathrm{N}=4, \mathrm{~N}=6)$ subcarriers. For simplicity, the parameter $R_{n}$ is set to $1 \mathrm{bit} / \mathrm{s}$ and variance $\sigma_{h}^{2}$ is 1.

To show the benefit of cooperative network coding, we simulate the link capacity and outage probability to valid the theoretical results.

First, Fig.2 presents the link capacity comparison of CT, CT_NC with DT as a basic benchmark. We can see that the CT_NC is about $11.8 \%$ higher than conventional CT scheme, while CT keeps a $32.5 \%$ higher than the normal direct transmission over two time slots. The advantage of CT over DT is due to the cooperative diversity over two uncorrelative channels. Moreover, CT_NC achieves both network coding diversity and cooperative diversity compared with CT, which brings the highest link capacity of the three schemes. We also see that the advantage keeps through the SNR axis.

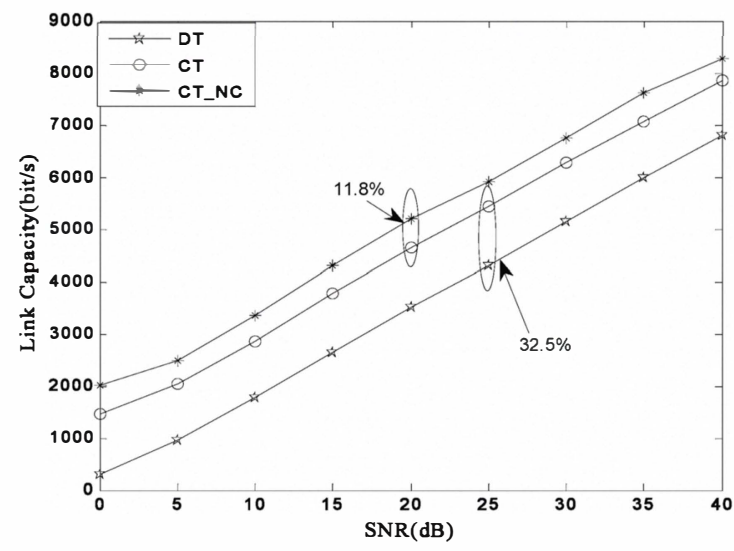

Figure 2. Capacity comparison with variance of SNR 


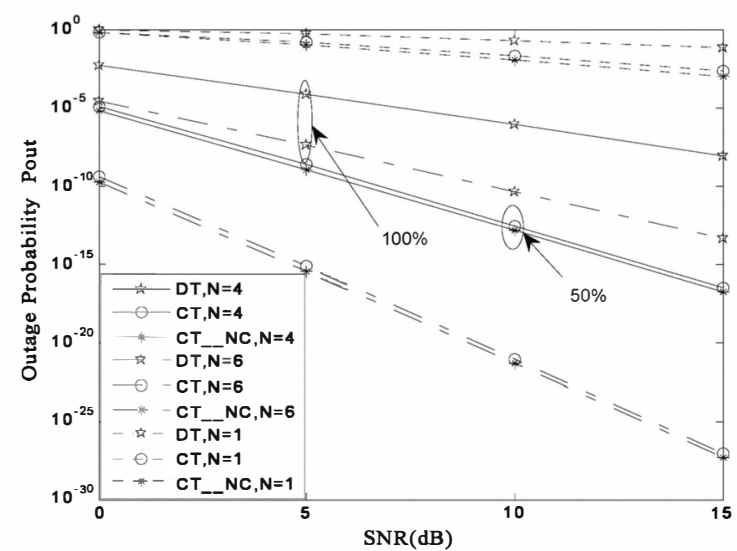

Figure 3. Outage probability comparison with variance of SNR

Then, we compare the outage probability comparision with the variance of SNR and subcarrier numbers in Fig.3. Taking $\mathrm{N}=4$ for example, the CT_NC improves the outage probability by $50 \%$ compared with $\mathrm{C} \overline{\mathrm{T}}$. This is because that the component data in the networking coded packets brings data redundancy, which ensures the successful transmission rate. Meanwhile, we can see that CT reduces $100 \%$ outage probability than DT. The reason is that we only considered one slot outage probability in the DT cases. If we use repetition coding for the two time slot, DT will achieve the same outage probability with CT. This is due to our previous assumption that subcarriers are allocated to guarantee the same outage probability for two users. We can also see the conclusion that the OFDMA reduces the outage probability at exponential trend by multiple orthogonal channels, which reinforces the expression (14) deduced from [13].

\section{CONCLUSIONS}

In this paper, we analyze two classical performance metrics for user cooperation with network coding scheme. Two closed formulation of capacity and outage probability for OFDMA based two users cooperation are presented. Compared with the DT and conventional CT, simulation results show the advantages of CT_NC. It provides $11.8 \%$ higher link capacity than CT cases averagely; meanwhile, it obtains $50 \%$ lower outage probability. Therefore, CT_NC is much more powerful in both the system throughput and robustness due to the network coding diversity than the direct transmission scheme and conventional user cooperative protocol.

\section{ACKNOWLEDGMENT}

This work is supported by National High-Tech Research and Development Plan of China under Grant No. 2009AA01Z206, the National Natural Science Foundation of China under Grant No. 6097108 and National International Science and Technology Cooperation Project under Granted NO.2008DFA12090.

\section{REFERENCES}

[1] Minghai Feng, Xiaoming She, Lan Chen, "Enhanced bidirectional relaying schemes for multi-hop communications," 2008.IEEE Global Telecommunications Conference, pp.1-6, 2008.

[2] D.Budimir, B.N. Shelkovnikov, "CAD for broadband wireless access design", TELSIKS 2001. 5th International Conference on Teleconmmunications in Modern Satellite, Cable and Broadcasting Service, vol. 2, pp. 525-528, 2001.

[3] Bin Lin, Pin-Han Ho, "Optimal Relay Station Placement in IEEE 802.16j Networks”, IWCMC’07, August 12-16, 2007.

[4] Lei Xiao, T. Fuja, J. Kliewer, D. Costello, "A network coding approach to cooperative diversity," IEEE Transactions on Information Theory, vol 53, pp. 3714-3722, 2007.

[5] Zhiguo Ding, K.K. Leung, D.L. Goeckel, D. Towsley, "On the Study of Network Coding with Diversity," IEEE Transactions on Wireless Communications, vol. 8, pp. 1247-1259, 2009.

[6] G.D. Menghwar, C.F. Mecklenbrauker, "Outage Performance of Two Users Cooperative Network Coding," $9^{\text {th }}$ International Symposium on Communications and Information Technology, pp. 1180-1184, 2009.

[7] Cong Peng, Qian Zhang, Ming Zhao, Yan Yao, Weijia Jia, "On the Performance Analysis of Network-Coded Cooperation in Wireless Networks," IEEE Transactions on Wireless Communications, vol. 7, pp. 3090-3097, 2008.

[8] P. Larsson, N. Johansson, K.-E. Sunell, "Coded Bi-directional Relaying," IEEE $63^{\text {rd }}$ Vehicular Technology Conference, vol. 2, pp. 851855,2006

[9] D.H. Woldegebreal, S. Valentin, H. Karl, "Incremental network coding in cooperative transmission wireless networks," IEEE $68^{\text {th }}$ Vehicular Technology Conference, pp. 1-5, 2008.

[10] A. Nosratinia, T.E. Hunter, A. Hedayat, "Cooperative communication in wireless networks,” IEEE Communications Magazine, vol. 42, pp. 74-80, 2004.

[11] Truman Chiu-Yam Ng, Wei Yu, "Joint optimization of relay strategies and resource allocations in cooperative cellular networks," IEEE Journal on Selected Areas in Communication, vol. 25, pp. 328-339, 2007.

[12] Bo Bai, Wei Chen, Zhigang Cao, K. Ben Letaief, "High-Order Analysis of Outage Probability in OFDMA Wireless Networks," IEEE Global Telecommunications Conference, pp. 1-6, 2009.

[13] Bo Bai, Wei Chen, Zhigang Cao, K. Ben Letaief, "Max-Matching Diversity in OFDMA Systems," IEEE Transactions on Communications, vol 58, pp. 1161-1171, 2010. 